\title{
Bone morphogenetic protein 2 alleviated intervertebral disc degeneration through mediating the degradation of ECM and apoptosis of nucleus pulposus cells via the PI3K/Akt pathway
}

\author{
YANLIN TAN $^{1,2}$, XINGWANG YAO $^{1}$, ZHEHAO DAI $^{1}$, YUNHUA WANG $^{2}$ and GUOHUA LV ${ }^{1}$ \\ Departments of ${ }^{1}$ Spinal Surgery and ${ }^{2}$ Positron Emission Tomography/Computed Tomography Center, \\ The Second Xiangya Hospital, Central South University, Changsha, Hunan 410011, P.R. China
}

Received January 29, 2018; Accepted October 22, 2018

DOI: $10.3892 /$ ijmm.2018.3972

\begin{abstract}
The present study aimed to explore the underlying mechanisms of bone morphogenetic protein 2 (BMP2) in alleviating intervertebral disc degeneration (IDD). A rat puncture IDD model was constructed, and the rats were randomly divided into six groups: Control; IDD (model); IDD+PBS [containing $10^{10}$ adeno-associated virus serotype 2 (AAV)]; and IDD + AAV2-BMP2 $\left(10^{6}, 10^{8}\right.$ and $\left.10^{10}\right)$. IL-1 $\beta$ was used to treat primary nucleus pulposus (NP) cells to mimic IDD in vitro. The effects of BMP2 in IDD were determined by magnetic resonance imaging (MRI), hematoxylin and eosin staining and Alcian Blue staining in vivo. The levels of collagen II, aggrecan, transcription factor SOX9 (SOX9) and matrix metalloproteinase 13 (MMP-13) were examined using western blot analysis and reverse transcription quantitative polymerase chain reaction (RT-qPCR) in NP tissues and cells. The expression of C-telopeptide of type II collagen (CTX-II) in the sera or cell supernatants was determined by ELISA. In addition, the levels of phosphorylation of phosphoinositide 3-kinase (PI3K) and protein kinase B (Akt), and the levels of apoptosis-associated proteins and apoptosis ratio of NP cells were also determined by western blot analysis and flow cytometry, respectively. LY29400, an inhibitor of PI3K, was used to additionally confirm the signal pathway mechanism of BMP2 treatment in IDD. BMP2 significantly extended the interval between discs and alleviated the fibrous ring rupture and the decrease in the levels of glycoproteins in IDD rats, as determined by MRI and histological staining. Additionally, BMP2 treatment significantly upregulated the levels of collagen II, aggrecan and SOX9, but downregulated the levels of MMP-13
\end{abstract}

Correspondence to: Dr Yanlin Tan, Department of Spinal Surgery, The Second Xiangya Hospital, Central South University, 139 Renmin Middle Road, Changsha, Hunan 410011, P.R. China E-mail: tanyanlin@csu.edu.cn

Key words: bone morphogenetic protein 2, intervertebral disc degeneration, nucleus pulposus cell, apoptosis, phosphoinositide 3-kinase/protein kinase B and CTX-II in IDD rats and NP cells in a dose-dependent manner. Concurrently, recombinant human (rh)BMP2 pretreatment also significantly decreased the apoptosis ratio of interleukin (IL)-1 $\beta$-treated NP cells via downregulating the level of cleaved caspase- 3 and upregulating the level of uncleaved poly (adenosine 5'-diphosphate-ribose) polymerase. It was demonstrated that rhBMP2 also significantly decreased the inflammatory response in NP tissues and cells, based on levels of IL-6, TNF- $\alpha$ and IL-10. In addition, rhBMP2 inhibited cell apoptosis via upregulating the phosphorylation levels of the PI3K/Akt signaling pathway, and LY29400 pretreatment inhibited the effects of BMP2 in IL-1 $\beta$ treated NP cells. BMP2 alleviated IDD via the PI3K/Akt signaling pathway by inhibiting NP cell apoptosis and decreasing the levels of matrix proteins.

\section{Introduction}

Intervertebral discs are the primary component of the human spine, which provides mechanical support and spinal motion for daily activities (1). Intervertebral disc degeneration (IDD) is an important contributor to neck, back and radicular pain, and a leading cause of disability worldwide (2). Commonly, IDD is characterized by disc space narrowing, water content reduction in the nucleus pulposus (NP) tissues, the appearance of fibrosis that is reflected in an increase in thickness and spacing of collagen fibers, and in the secretion of proinflammatory cytokines $(3,4)$. Specifically, NP tissues destruction and deceleration of the synthesis of disc components are the two primary causes of IDD pathogenesis (5). Several factors have been demonstrated to contribute to IDD, including hip osteoarthritis (6), obesity (7) and genetic factors, but the exact mechanisms of IDD remain unclear. With the development of medical technology in previous years, several methods have been explored to treat IDD, including anti-inflammatory medication, analgesics, physical therapy and surgery, but the outcome is unsatisfactory $(8,9)$. Therefore, it is necessary to explore novel therapeutic methods to improve the treatment of IDD.

The extracellular matrix (ECM) primarily consists of proteoglycans, aggrecan, collagens and matrix metalloproteinases (MMPs) in NP. Degradation of the ECM, particularly 
collagen II and aggrecan, is considered to be an important cause of IDD (10). Collagen II is an important component of ECM, and its expression is significantly downregulated during the IDD process $(11,12)$. Mutation of collagen type II alpha 1 chain, the encoding gene of collagen II, is commonly identified in spondyloepiphyseal dysplasia congenita, knee osteoarthritis, kniest dysplasia and type II collagenopathies (13-15). C-telopeptide of type II collagen (CTX-II) is a degradation product of collagen II, and is usually upregulated in the sera of patients with osteoarthritis (16). Yang et al (17) suggested that E2 inhibited aberrant apoptosis of NP cells via upregulating the expression of collagen II and aggrecan, and downregulating the expression of MMP-13. MMP-13 has been identified to degrade collagen II in IDD (18). Considering these variations in ECM, therapeutics for degenerative diseases may be developed by increasing the synthesis of collagen II and aggrecan, while inhibiting the expression of MMPs.

Bone morphogenetic proteins (BMPs) belong to the transforming growth factor $\beta$ (TGF- $\beta$ ) family, and are commonly known to function as underlying regulators of bone and cartilage formation (19). Specifically, BMP2 has been widely demonstrated to be involved in the regulation of diseases, including non-union fractures, osteoporosis and spinal fusion, as reviewed in previous literatures $(20,21)$. BMP2 was also suggested to protect the growth and decrease the apoptosis of NP cells (22), but the exact mechanism remains unclear. An animal model demonstrated that combination therapy of BMP2 and a receptor activator of nuclear factor $\kappa \mathrm{B}$ ligand inhibitor promoted bone healing in a critical-sized femoral defect mouse model (23). Additionally, BMP2 inhibited the growth and metastasis of hepatocellular carcinoma by inhibiting the phosphoinositide 3-kinase (PI3K)/protein kinase B (Akt) pathway, which serves an important role in osteogenesis $(24,25)$. Concurrently, activation of the PI3K/Akt signaling pathway may protect against IDD via enhancing the production of aggrecans and collagen II (26). However, whether BMP2 alleviates IDD via the PI3K/Akt pathway remains unclear.

In light of the aforementioned data, the present study aimed to explore the underlying mechanisms of BMP2 in alleviating IDD in a rat model and NP cells in vitro. The present study indicated that BMP2 alleviated IDD via activating the PI3K/Akt signaling pathway to inhibit NP cells apoptosis and the decrease in matrix protein degradation, particularly aggrecans and collagen II. These data may provide novel insights into the understanding and treatment of IDD in clinical settings.

\section{Materials and methods}

Preparation of adeno-associated virus serotype 2 (AAV)-BMP2 adenovirus. The 293T cells were purchased from the Cell Bank of the Chinese Academy of Sciences (Shanghai, China), and maintained in Dulbecco's modified Eagle's medium (DMEM; Gibco; Thermo Fisher Scientific, Inc., Waltham, MA, USA) containing $10 \%$ fetal bovine serum (FBS; Gibco; Thermo Fisher Scientific, Inc.) in a humidity incubator at $37^{\circ} \mathrm{C}$ with $5 \% \mathrm{CO}_{2}$. Subsequently, $1 \mu \mathrm{g}$ recombinant plasmid pDC315-BMP2 (Shanghai GenePharma Co., Ltd., Shanghai, China) was co-transfected for $4 \mathrm{~h}$ with $10 \times 10^{6}$ pfu pBGHE3 (Shanghai GenePharma Co., Ltd.) into 293T cells using Lipofectamine $2000^{\circledR}$ (Invitrogen; Thermo Fisher Scientific, Inc.) to obtain the recombinant BMP2 adenovirus. Following this, the titer of viral particles was estimated by dot blot assay as described previously (27) and purified stocks were maintained at $\sim 10^{12}$ particles $/ \mathrm{ml}$.

Construction of IDD model and grouping. The experimental procedures of the present study were approved by the Second Xiangya Hospital, Central South University (Changsha, China). All the procedures involving animals and their care were performed in accordance with the Guidelines for the Care and Use of Laboratory Animals of the Second Xiangya Hospital, Central South University. A total of 48 male Lewis rats aged 13-14 weeks were purchased from Shanghai SLAC Laboratory Animal Co., Ltd. (Shanghai, China), and housed in a 12-h light/dark cycle sterile room at $\sim 23^{\circ} \mathrm{C}$ with access to food and water ad libitum. Rats were randomly divided into six groups ( $\mathrm{n}=8$ per group): Control; punctured (model); puncture + PBS injection (5 $\mu \mathrm{l}$, containing $\left.10^{10} \mathrm{AAV}\right)$; punctured $+10^{6}$ AAV-BMP2 $(5 \mu \mathrm{l})$; punctured + $10^{8}$ AAV-BMP2 $(5 \mu \mathrm{l})$; and punctured $+10^{10} \mathrm{AAV}-\mathrm{BMP} 2(5 \mu \mathrm{l})$. The IDD model was generated by using the annulus fibrosus needle puncture method (28). Briefly, rats were anesthetized intraperitoneally with sodium pentobarbital $(30 \mathrm{mg} / \mathrm{kg})$. A $18 \mathrm{G}$ syringe needle was inserted into the $\mathrm{C} 6-\mathrm{C} 7$ and $\mathrm{C} 8-\mathrm{C} 9$ discs in the vertical direction with $5 \mathrm{~mm}$ depth. Following full penetration, the needle was rotated $360^{\circ}$ and held for $20 \mathrm{sec}$. For the control group, the discs were exposed, but not punctured. For the later groups, PBS (containing $10^{10} \mathrm{AAV}$ ) or AAV-BMP2 was injected into punctured location on week 2 subsequent to puncture. All rats were sacrificed following 8 weeks by inhalation of $7.5 \%$ isoflurane, and the NP tissues and blood samples were collected.

Magnetic resonance imaging (MRI) acquisition. A total of 2 months after surgery, rats were anesthetized as aforementioned to limit movement during the MRI examination. Images were obtained using a 3.0 T MRI machine (Philips Healthcare, Amsterdam, The Netherlands) with a dedicated coil for small animals. Following this, the tails of the rats were immersed in $0.1 \mathrm{M} \mathrm{CuSO}_{4}$ solution in a tube, to increase the contrast of image. Subsequently, a 2-D spin echo-dual echo sequence was performed with the following parameters: Repetition time $=9,000 \mathrm{~ms}$, flip angle $=90^{\circ}$, echo times $=16$ and $80 \mathrm{~ms}$, slice thickness $=0.6$, number of averages $=2$, field of view $=40 \times 40 \mathrm{~mm}^{2}$, and in-plane resolution $=0.1 \mathrm{~mm}$ with 30 sagittal slices. The disc signal intensity was calculated by using the $\mathrm{T} 2$-weighted image (echo time $=80 \mathrm{~ms}$ ) to monitor the disc hydration. Then, the mean signal intensity (brightness) in the control disc was used as reference for the signal intensity of the injured discs in each group. Therefore, the value of normalized intensity for the injured discs ranged from $0-1$, as described previously (28).

Histological analysis. Following MRI examination, rats were sacrificed and the whole discs with the vertebrae adjacent to the punctured $\mathrm{C} 6-\mathrm{C} 7$ and $\mathrm{C} 8-\mathrm{C}$, and non-punctured $\mathrm{C} 7-\mathrm{C} 8$ were isolated, fixed with $4 \%$ paraformaldehyde at $4^{\circ} \mathrm{C}$ for $24 \mathrm{~h}$, decalcified in $10 \%$ EDTA for 30 days, embedded in paraffin 
and cut into $5-\mu \mathrm{m}$ thick slices. Then, the slices were dehydrated with an ethanol gradient $(70,90 \%)$ and stained for $30 \mathrm{~min}$ at room temperature with hematoxylin and eosin (H\&E; Nanjing Jiancheng Bioengineering Institute, Nanjing, Jiangsu, China) according to manufacturer's protocols. Concurrently, parts of the slices were stained for $30 \mathrm{~min}$ at room temperature with 1\% Alcian Blue (pH 2.5; Newcomer Supplu Inc., Middleton, WI, USA) following dehydration. Following this, sections were permeabilized with $100 \%$ xylene and sealed with neutral resins. Subsequently, the stained slides were analyzed under a light microscope at x400 magnification (Nikon E800 microscope, Nikon Instruments Inc., Melville, NY, USA).

NP cells isolation and treatment. NP cells were isolated as described previously (29,30). Following isolation, NP cells were resuspended in DMEM medium supplied with $10 \%$ FBS, $100 \mu \mathrm{g} / \mathrm{ml}$ streptomycin (Invitrogen; Thermo Fisher Scientific, Inc.), and $100 \mathrm{U} / \mathrm{ml}$ penicillin (Invitrogen; Thermo Fisher Scientific, Inc.) at $37^{\circ} \mathrm{C}$ in a humidified atmosphere with $5 \% \mathrm{CO}_{2}$. The medium was changed every two days, and the second passage was used for subsequent experiments.

For treatment, NP cells were first treated with different concentrations of recombinant human BMP2 (rhBMP2; 0, 25, $50,100,200,400$ and $800 \mathrm{ng} / \mathrm{ml}$ ) to assess the effects of rhBMP2 on cell proliferation, and for the selection of appropriate concentrations for subsequent experimentation. NP cells were treated with $10 \mathrm{ng} / \mathrm{ml}$ interleukin $1 \beta$ (IL-1 $\beta$; Sigma-Aldrich; Merck KGaA, Darmstadt, Germany) to imitate the IDD model in vitro. For subsequent experiments, cells were pretreated with $10 \mu \mathrm{M}$ LY294002 (PI3K signal pathway inhibitor) for $1 \mathrm{~h}$ at $37^{\circ} \mathrm{C}$ to verify the mechanism of the signal pathway.

MTT assay. NP cells were seeded in 96-well plates at a density of $1.0 \times 10^{3} /$ well and cultured with complete DMEM medium containing different concentrations of rhBMP2 $(0,25,50,10$ $0,200,400$ and $800 \mathrm{ng} / \mathrm{ml})$. Then, cells were cultured for 24 and $48 \mathrm{~h}$. Subsequently, $20 \mu \mathrm{l}$ MTT solution $(5 \mathrm{mg} / \mathrm{ml})$ was added into each well and plates were incubated at $37^{\circ} \mathrm{C}$ for $4 \mathrm{~h}$. Then, the supernatant was discarded and $150 \mu \mathrm{l}$ dimethyl sulfoxide was added into each well, and plates were agitated at room temperature for $10 \mathrm{~min}$. Following this, the optical density (OD) values were determined at $570 \mathrm{~nm}$.

Apoptosis assay. The apoptosis of cells was determined by flow cytometry with Kaluza 2017 Analysis Software 1.3 (Beckman Coulter, Inc., Brea, CA, USA) following staining with fluorescein isothiocyanate (FITC)-conjugated Annexin V and propidium iodide (PI; Beyotime Institute of Biotechnology, Haimen, China). Briefly, $1 \times 10^{6}$ cells were seeded in 6-well plates with DMEM containing $10 \% \mathrm{FBS}$ overnight at $37^{\circ} \mathrm{C}$. Then, cells were treated with rhBMP2 and IL- $1 \beta$ as aforementioned for $48 \mathrm{~h}$. Following this, the cells were stained using the FITC Annexin V/PI apoptosis Detection kit I (BD Pharmingen; BD Biosciences, Franklin Lakes, NJ, USA) for $20 \mathrm{~min}$ at room temperature according to the manufacturer's protocol. Subsequently, the levels of apoptosis of the cells were detected using flow cytometry. Annexin $\mathrm{V}^{+}$cells were considered early apoptotic cells, Annexin $\mathrm{V}^{+} / \mathrm{PI}^{+}$cells were considered end-stage apoptotic cells and $\mathrm{PI}^{+}$cells were considered necrotic cells.
Reverse transcription quantitative polymerase chain reaction $(R T-q P C R)$. Total RNA was extracted from rat NP tissues and cells using TRIzol ${ }^{\circledR}$ reagent (Life Technologies; Thermo Fisher Scientific, Inc.) according to manufacturer's protocol. Following this, the mRNA was reverse transcribed into cDNA using a PrimeScript RT reagent kit (Takara Biotechnology Co., Ltd., Dalian, China) according to manufacturer's protocol. Then, the cDNA was used as the template to perform qPCR (BeyoFast $^{\mathrm{TM}}$ SYBR Green qPCR Mix; Beyotime Institute of Biotechnology) with the following thermocycler conditions: $95^{\circ} \mathrm{C}$ for $5 \mathrm{sec}$, then 45 cycles comprising denaturing at $95^{\circ} \mathrm{C}$ for $30 \mathrm{sec}$, annealing at $60^{\circ} \mathrm{C}$ for $20 \mathrm{sec}$ and extending at $72^{\circ} \mathrm{C}$ for $30 \mathrm{sec}$ on an ABI 7500 Real-time PCR System (Applied Biosystems; Thermo Fisher Scientific, Inc.). The primers used are listed in Table I. Gene expression levels were normalized to GAPDH, and were analyzed using the $2^{-\Delta \Delta C q}$ method (31).

ELISA assay. The level of CTX-II in rat serum and culture supernatant following $1,000 \mathrm{x} \mathrm{g}$ centrifugation at room temperature for $5 \mathrm{~min}$ was detected using CTX ELISA kit (cat. no. E-EL-M0368c; Ela bscience, Wuhan, China) according to the manufacturer's protocols.

Western blot analysis. Total proteins in NP tissues and cells were isolated by using the radioimmunoprecipitation assay lysis buffer (Beyotime, Nanjing, China) containing 1X protease inhibitor cocktail and 1X PhosStop (both, Roche Diagnostics, Indianapolis, IN, USA). The concentration of protein was determined by using a BCA protein assay kit (Thermo Fisher Scientific, Inc.) according to the manufacturer's protocol. Then, the protein extracts were boiled with equal volumes of loading buffer (Beyotime Institute of Biotechnology) for $10 \mathrm{~min}$. Following this, $15 \mu \mathrm{g}$ total protein was loaded onto $10 \%$ SDS-PAGE gels for electrophoresis, and then electro-transferred onto polyvinylidene fluoride membranes. Following this, the membranes were blocked with $5 \%$ non-fat milk solution in TBS-Tween 20 at room temperature for $1 \mathrm{~h}$. Subsequently, membranes were incubated with the following anti-rat primary antibodies $(1: 1,000)$ at $4^{\circ} \mathrm{C}$ overnight: Cleaved caspase-3 (cat. no. 9662; Cell Signaling Technology, Inc., Danvers, MA, USA), PARP (cat. no. 9542; Cell Signaling Technology, Inc.), collagen II (cat. no. ab34712; Abcam, Cambridge, United Kingdom), aggrecan (cat. no. ab3778; Abcam), transcription factor SOX9 (SOX9; cat. no. ab185966; Abcam), MMP-13 (cat. no. 94808; Cell Signaling Technology, Inc.), phosphorylated (p)-PI3K (cat. no. 4228; Cell Signaling Technology, Inc.), PI3K (cat. no. 4249; Cell Signaling Technology, Inc.), p-AKT (cat. no. 4685; Cell Signaling Technology, Inc.), AKT (cat. no. 4060; Cell Signaling Technology, Inc.) and GAPDH (cat. no. ab8245; Abcam). Following this, the membranes were incubated with horseradish peroxidase-conjugated secondary antibodies (anti-rabbit, cat. no. ab150077; anti-mouse, cat. no. ab6785; both, 1:5,000; both, Abcam) at room temperature for $1 \mathrm{~h}$. The protein bands in the membranes were then visualized using the enhanced chemiluminescent method (EMD Millipore, Billerica, MA, USA), and quantified using Quantity One software 4.2.1 (Bio-Rad Laboratories, Inc., Hercules, CA, USA). GAPDH was used as the internal control to normalize the expression of the proteins. 
Table I. Primers for reverse transcription quantitative polymerase chain reaction.

\begin{tabular}{|c|c|c|}
\hline Gene & Primers & Sequences $\left(5^{\prime}-3^{\prime}\right)$ \\
\hline \multirow[t]{2}{*}{ Collagen-II-h } & Forward & GGCAATAGCAGGTTCACGTACA \\
\hline & Reverse & CGATAACAGTCTTGCCCCACTT \\
\hline \multirow[t]{2}{*}{ SOX9-h } & Forward & AGCGAACGCACATCAAGAC \\
\hline & Reverse & CTGTAGGCGATCTGTTGGGG \\
\hline \multirow[t]{2}{*}{ Aggrecan-h } & Forward & TCCACAAGGGAGAGAGGGTA \\
\hline & Reverse & GTAGGTGGTGGCTAGGACGA \\
\hline \multirow[t]{2}{*}{ MMP-13-h } & Forward & GGCTCCGAGAAATGCAGTCTTTCTT \\
\hline & Reverse & ATCAAATGGGTAGAAGTCGCCATGC \\
\hline \multirow[t]{2}{*}{ IL-6-h } & Forward & ATGAACTCCTTCTCCACAAGC \\
\hline & Reverse & CTACATTTGCCGAAGAGCCCTCAGGCTGGACTG \\
\hline \multirow[t]{2}{*}{ IL-10-h } & Forward & AGGGCACCCAGTCTGAGAACA \\
\hline & Reverse & CGGCCTTGCTCTTGTTTTCAC \\
\hline \multirow[t]{2}{*}{ TNF- $\alpha-h$} & Forward & ATGAGCACTGAAAGCATGATC \\
\hline & Reverse & TCACAGGGCAATGATCCCAAAGTAGACCTGCCC \\
\hline \multirow[t]{2}{*}{ GAPDH-h } & Forward & AAGGTCGGAGTCAACGGATTT \\
\hline & Reverse & AGATGATGACCCTTTTGGCTC \\
\hline \multirow[t]{2}{*}{ Collagen-II-r } & Forward & ACGCTCAAGTCGCTGAACAA \\
\hline & Reverse & TCAATCCAGTAGTCTCCGCTCT \\
\hline \multirow[t]{2}{*}{ SOX9-r } & Forward & TCCAGCAAGAACAAGCCACA \\
\hline & Reverse & CGAAGGGTCTCTTCTCGCTC \\
\hline \multirow[t]{2}{*}{ Aggrecan-r } & Forward & TCCAAACCAACCCGACAAT \\
\hline & Reverse & TCTCATAGCGATCTTTCTTCTGC \\
\hline \multirow[t]{2}{*}{ MMP-13-r } & Forward & ATGCAGTCTTTCTTCGGCTTAG \\
\hline & Reverse & ATGCCATCGTGAAGTCTGGT \\
\hline \multirow[t]{2}{*}{ IL-6-r } & Forward & CCTCTGGTCTTCTGGAGTACC \\
\hline & Reverse & АCTCCTTCTGTGACTCCAGC \\
\hline \multirow[t]{2}{*}{ IL-10-r } & Forward & ATAACTGCACCCACTTCCCA \\
\hline & Reverse & GGGCATCACTTCTACCAGGT \\
\hline \multirow[t]{2}{*}{ TNF- $\alpha-r$} & Forward & ATGAGCACAGAAAGCATGA \\
\hline & Reverse & AGTAGACAGAAGAGCGTGGT \\
\hline \multirow[t]{2}{*}{ GAPDH-r } & Forward & GGAAAGCTGTGGCGTGAT \\
\hline & Reverse & AAGGTGGAAGAATGGGAGTT \\
\hline
\end{tabular}

h, human; r, rat; SOX9, Transcription factor SOX9; MMP-13, matrix metalloproteinase; IL, interleukin; TNF- $\alpha$, tumor necrosis factor $\alpha$.

Statistical analysis. In the present study, all data are presented as mean \pm standard deviation. Statistical analyses were performed using GraphPad Prism 5 (GraphPad Software, Inc., La Jolla, CA, USA). Statistical evaluation was performed using Student's t-test (two-tailed) between two groups, or one-way analysis of variance followed by Tukey's post hoc test for multiple comparisons. $\mathrm{P}<0.05$ was considered to indicate a statistically significant difference.

\section{Results}

BMP2 treatment alleviates IDD in rats. The pathogenic symptoms of IDD in each group were determined using MRI. Compared with the control group, the interval between punctured discs was significantly decreased in the IDD rat model, as indicated by the blue arrows, while AAV2-BMP2 treatment markedly inhibited the decrease in intervals between punctured discs in the IDD model in a dose-dependent manner (Fig. 1A). Additional H\&E assays revealed that there were clearly intact discs with well-opposed lamellae and unbroken annulus fibrosus with clearly-defined borders in the control group, but the IDD model group presented severe lamellar disorganization and broken annulus fibrosus, as indicated by the red arrows. AAV2-BMP2 treatment markedly alleviated the disorganization of lamellae and protected the annulus fibrosus structure of the discs (Fig. 1B). Alcian Blue staining indicated that the secretion of glycoproteins was significantly downregulated in the IDD model, and that AAV2-BMP2 injection attenuated this downregulation (Fig. 1B). All these data indicate that BMP2 may alleviate the symptoms of IDD in vivo.

BMP2 treatment regulates the levels of IDD-associated biomarkers and inflammatory cytokines. Following sacrifice, the levels of collagen II, aggrecan, SOX9, MMP-13 and CTX-II 
A

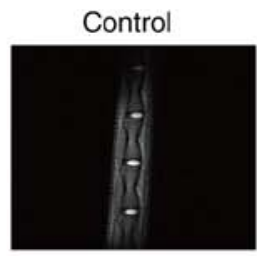

B

$\mathrm{H} \& \mathrm{E}$

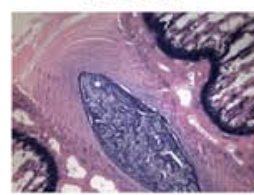

Control

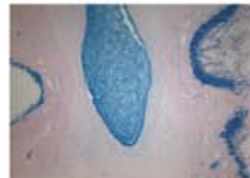

IDD

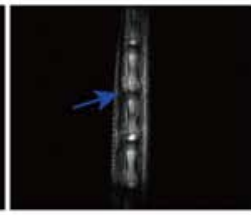

IDD

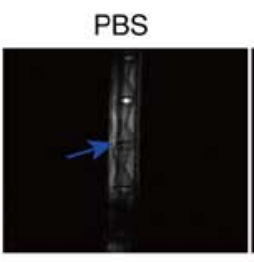

PBS

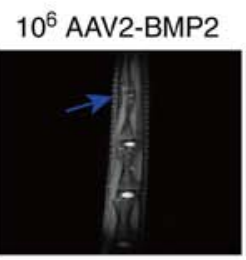

$10^{6}$ AAV2-BMP2

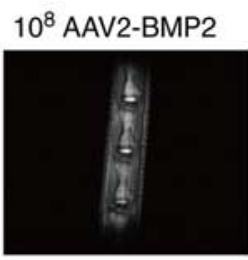

$10^{8}$ AAV2-BMP2

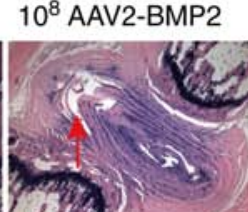

$10^{10}$ AAV2-BMP2
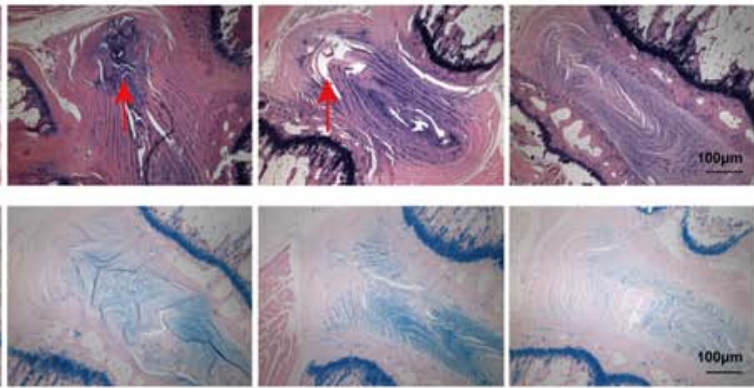

Figure 1. BMP2 alleviates IDD in rats. (A) IDD in rats determined using magnetic resonance imaging. (B) Histological analysis of intervertebral disc tissues determined by using H\&E and Alcian Blue staining. Blue arrows indicate the decreased interval between punctured discs. Red arrows indicate the unbroken annulus fibrosus. IDD, intervertebral disc degeneration; H\&E, hematoxylin and eosin; AAV2-BMP2, adeno-associated virus serotype 2-bone morphogenic protein 2.

in NP tissues of each group were determined by RT-qPCR. As a result, the levels of collagen II, aggrecan and SOX9 were significantly downregulated in the IDD group compared with the control group, while BMP2 overexpression significantly attenuated these changes in IDD in a dose-dependent manner (Fig. 2A). However, the serum levels of MMP-13 in NP tissues and CTX-II were significantly increased in IDD group compared with the control group, and overexpression of BMP2 significantly inhibited the upregulation of MMP-13 and CTX-II in a dose-dependent manner (Fig. 2A and B). IL-6, TNF- $\alpha$ and IL-10 levels in NP tissues were additionally examined using RT-qPCR. It was identified that BMP2 significantly inhibited the mRNA levels of proinflammatory cytokines IL-6 and TNF- $\alpha$, but significantly increased the levels of anti-inflammatory cytokine IL-10 mRNA, in a dose-dependent manner (Fig. 2C). Taken together, these results suggest that BMP2 may suppress the degradation of the extracellular matrix and inflammatory response during IDD.

rhBMP2 treatment inhibits the apoptosis of NP cells during $I D D$. To explore the underlying effects of BMP2 on the alleviation of IDD in vitro, an IDD cell model was constructed using IL-1 $\beta$, and the viability of NP cells following treatment with rhBMP2 was determined. As a result, rhBMP2 markedly inhibited the survival ability of NP cells when being treated with high concentrations at 24 and $48 \mathrm{~h}$ (Fig. 3A). Therefore, the lower concentrations $(25,50$ and $100 \mathrm{ng} / \mathrm{ml})$ were selected for subsequent experiments. Compared with the control group, IL-1 $\beta$ treatment significantly increased the levels of apoptosis of NP cells, and rhBMP2 pretreatment markedly attenuated the apoptosis ratio induced by IL-1 $\beta$ in the NP (Fig. 3B and C). Concurrently, the levels of apoptosis-associated proteins were also determined by western blot analysis. IL-1 $\beta$ treatment significantly increased the level of cleaved caspase-3 but decreased the level of uncleaved (full length) poly [adenosine 5'-diphosphate (ADP)-ribose] polymerase (PARP) compared with the control group, while rhBMP2 pretreatment significantly inhibited these changes in a dose-dependent manner (Fig. 3D and E). These results indicated that BMP2 inhibited apoptosis and promoted the survival ability of NP cells in a dose-dependent manner during the pathogenesis of IDD.

rhBMP2 attenuates the levels of IDD-associated proteins and inflammatory response induced by $I L-1 \beta$ in NP cells. The levels of collagen II, aggrecan, SOX9 and MMP-13 in cells, and levels of CTX-II in the supernatant were also determined in vitro. It was identified that the mRNA levels of collagen II, aggrecan and SOX9 were significantly downregulated, while the level of MMP-13 was significantly increased in the cells following treatment with IL-1 $\beta$. Additionally, rhBMP2 pretreatment significantly attenuated these changes in a dose-dependent manner (Fig. 4A). The western blot analysis results also indicated that IL-1 $\beta$ treatment markedly decreased the protein levels of collagen II, aggrecan and SOX9, but increased the level of MMP-13 in NP cells, while rhBMP2 treatment inhibited these changes in a dose-dependent manner (Fig. 4B and C). In addition, the ELISA assay results demonstrated that IL-1 $\beta$ significantly increased the level of CTX-II, and rhBMP2 pre-treatment suppressed this increase in a dose-dependent manner (Fig. 4D). IL-6, TNF- $\alpha$ and IL-10 levels in the NP cells were also examined using RT-qPCR, as demonstrated in Fig. 4E. It was identified that rhBMP2 exhibited similar effects on the levels of IL-6, TNF- $\alpha$ and IL-10 in NP tissues when its concentration was at 25 and $50 \mathrm{ng} / \mathrm{ml}$. When using high doses $(100 \mathrm{ng} / \mathrm{ml})$ of BMP2, no significant difference in the effect was observed. All of these data indicated that rhBMP2 may alleviate the decrease in matrix protein secretion and inhibit the inflammatory response in NP cells.

rhBMP2 inhibits the levels of extracellular matrix degradation and apoptosis of NP cells via the PI3K/Akt signaling pathway. To additionally reveal the mechanisms of extracellular matrix 


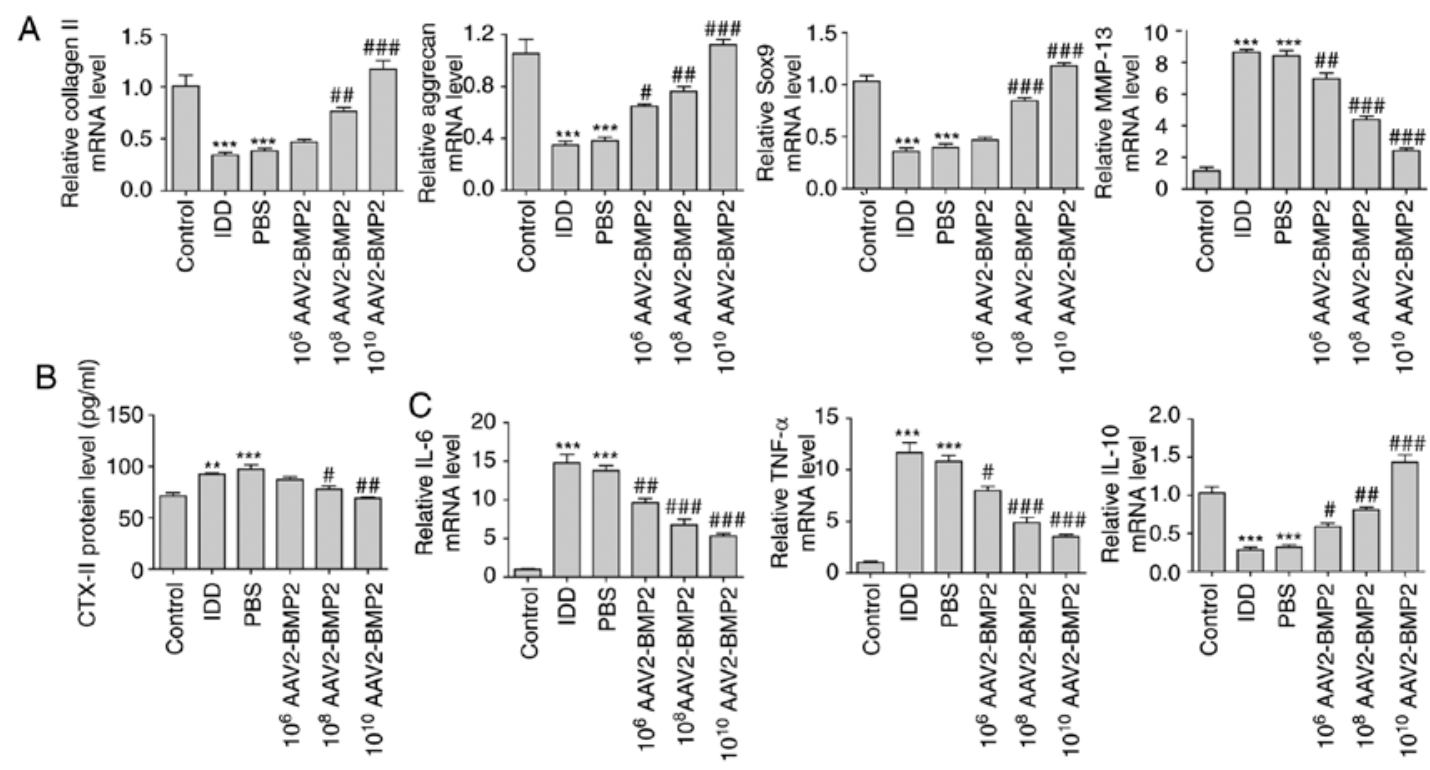

Figure 2. BMP2 treatment regulates the levels of IDD-associated biomarkers and inflammatory cytokines in NP tissues. (A) Relative mRNA levels of collagen II, aggrecan, SOX9 and MMP-13 in NP tissues determined using RT-qPCR. (B) Level of CTX-II in the serum of rats determined using ELISA. (C) Relative mRNA levels of IL-6, TNF- $\alpha$ and IL-10 determined using RT-qPCR. ${ }^{* *} \mathrm{P}<0.01$ and ${ }^{* * * *} \mathrm{P}<0.001$ vs. control group. ${ }^{\# P} \mathrm{P}<0.05,{ }^{\# \#} \mathrm{P}<0.01$ and ${ }^{\# \# \#} \mathrm{P}<0.001$ vs. IDD group. IDD, intervertebral disc degeneration; AAV2-BMP2, adeno-associated virus serotype 2-bone morphogenic protein 2; SOX9, transcription factor SOX9; MMP-13, matrix metalloproteinase 13; CTX-II, C-telopeptide of type II collagen; IL, interleukin; TNF- $\alpha$, tumor necrosis factor $\alpha$; RT-qPCR, reverse transcription quantitative polymerase chain reaction; NP, nucleus pulposus.
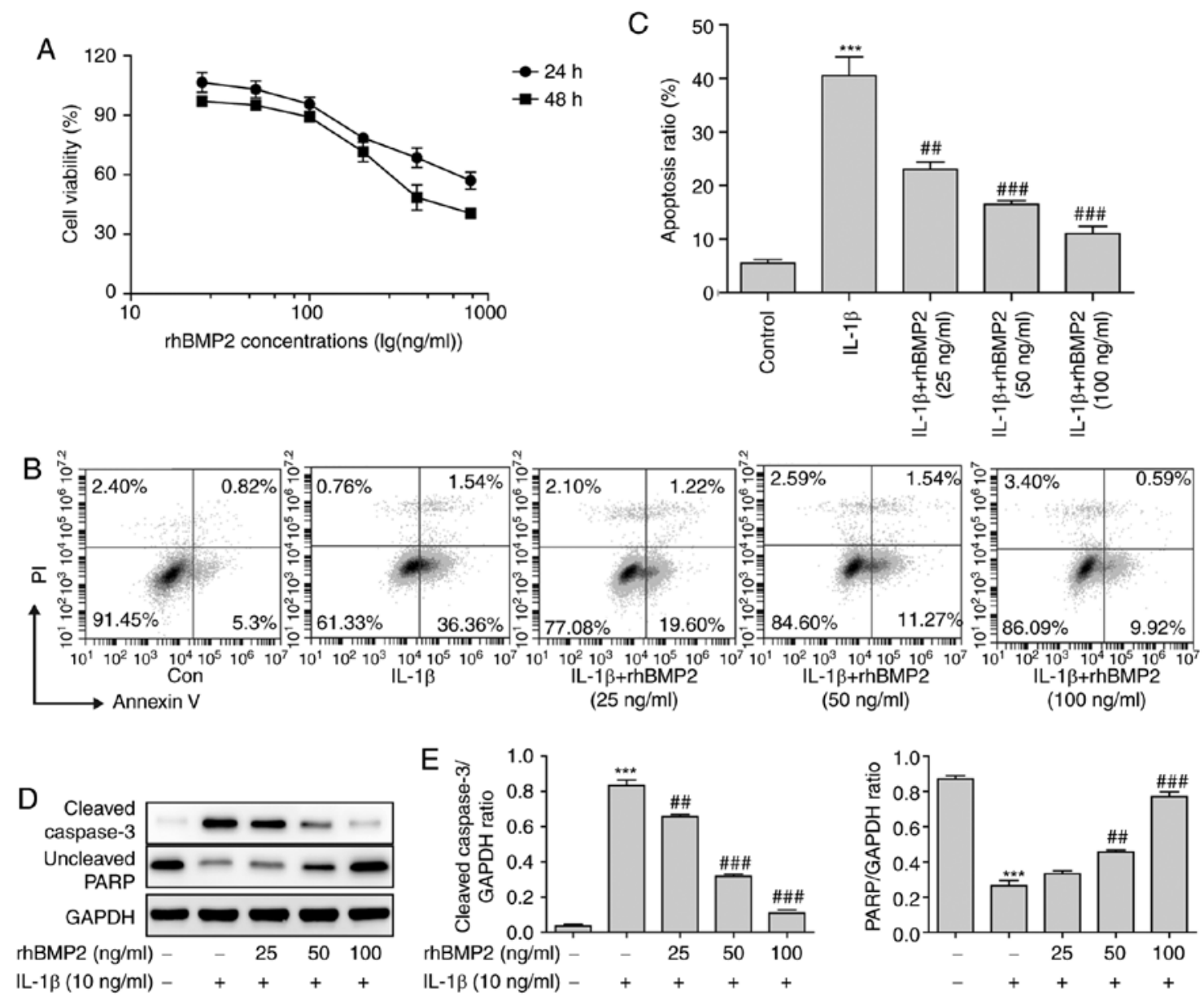

Figure 3. rhBMP2 treatment inhibits the apoptosis of NP cells during IDD. (A) Proliferation of NP cells following treatment with different concentrations of rhBMP2 determined by MTT assay. (B) Apoptosis of NP cells determined by flow cytometry. (C) Quantification of cell apoptosis. (D) Levels of cleaved caspase-3 and uncleaved PARP determined by western blot analysis. (E) Quantification of western blot analysis. ${ }^{* * * *} \mathrm{P}<0.001$ vs. control group. ${ }^{\# \#} \mathrm{P}<0.01$ and

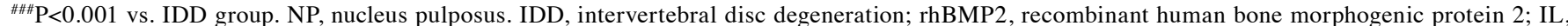
interleukin; PI, propidium iodide; Con, control; PARP, poly (adenosine 5'-diphosphate-ribose) polymerase; NP, nucleus pulposus. 


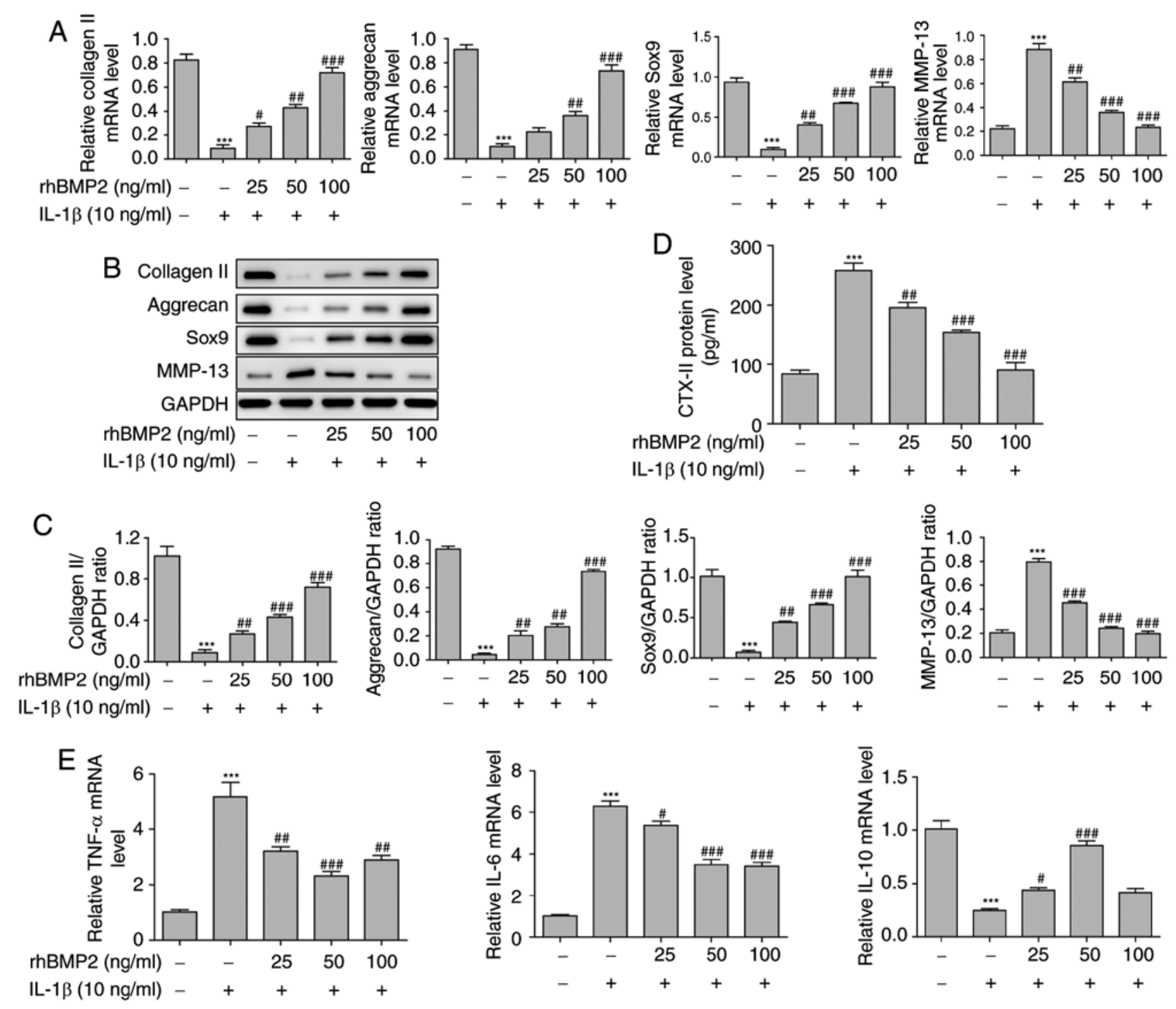

Figure 4. rhBMP2 attenuates the IDD-associated proteins and inflammatory response induced by IL-1 $\beta$ in NP cells. (A) Relative mRNA levels of collagen II, aggrecan, SOX9 and MMP-13 in NP cells determined by using RT-qPCR. (B) Levels of collagen II, aggrecan, SOX9 and MMP-13 in NP cells determined by western blot analysis. (C) Quantification of western blot analysis results of collagen II, aggrecan, SOX9 and MMP-13 in NP cells. (D) Level of CTX-II in the supernatant of NP cells determined by ELISA. (E) Relative mRNA levels of IL-6, TNF- $\alpha$ and IL-10 in NP cells determined by RT-qPCR. ${ }^{* * *} \mathrm{P}<0.001$ vs. control group. ${ }^{\#} \mathrm{P}<0.05,{ }^{\# \#} \mathrm{P}<0.01$ and ${ }^{\# \# \#} \mathrm{P}<0.001$ vs. the IL-1 $\beta$ treated group. SOX9, transcription factor SOX9; MMP-13, matrix metalloproteinase 13; CTX-II, C-telopeptide of type II collagen; IL, interleukin; TNF- $\alpha$, tumor necrosis factor $\alpha$; RT-qPCR, reverse transcription quantitative polymerase chain reaction; rhBMP2, recombinant human bone morphogenic protein 2; NP, nucleus pulposus.

degradation and apoptosis of NP cells during IDD, the proteins involved in the PI3K/Akt signaling pathway were examined. It was identified that the levels of total PI3K and Akt protein were not changed following treatment of NP cells with IL-1 $\beta$ and rhBMP2. However, the levels of phosphorylation of PI3K and Akt in NP cells were significantly decreased following IL-1 $\beta$ treatment. Additionally, rhBMP2 pretreatment significantly upregulated the levels of phosphorylation of PI3K and Akt in a dose-dependent manner (Fig. 5A and B). To additionally confirm whether the PI3K/Akt pathway was involved in IDD, LY294002, an inhibitor for PI3K, was utilized. Firstly, levels of phosphorylated Akt and total Akt in response to IL-1 $\beta$, rhBMP2 and LY294002 treatment we examined by western blot analysis to verify the function of LY294002 and the PI3K/Akt pathway, and the results indicated that LY294002 markedly inhibited the level of phosphorylation of Akt and reversed the effects induced by rhBMP2 (Fig. 5C and D). Additional results indicated that LY294002 treatment significantly attenuated the increase in collagen II, aggrecan and SOX9 levels, and the decrease in MMP-13 and CTX-II levels caused by rhBMP2 in $1 \mathrm{~L}-1 \beta$-treated NP cells (Fig. 5E and F). Pretreatment with LY294002 also markedly inhibited the decrease of cleaved caspase- 3 and the increase of uncleaved PARP induced by rhBMP2 in $1 \mathrm{~L}-1 \beta$-treated NP cells (Fig. 5G). Flow cytometry was also used to examine cell apoptosis following treatment with combinations of IL-1 $\beta$, rhBMP2 and LY294002. It was identified that IL-1 $\beta$ significantly increased the apoptosis rate of NP cells, but rhBMP2 significantly inhibited the IL-1 $\beta$-induced apoptosis rate of NP cells. Additionally, LY294002 reversed the effects of rhBMP2, and caused a significant increase in the apoptosis rate of rhBMP2-treated NP cells (Fig. 5H and 5I). All of these results 


\section{A}

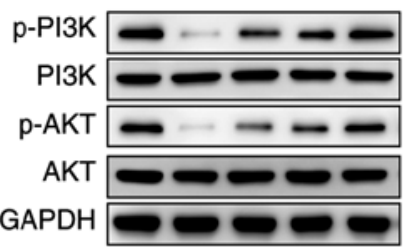

$\operatorname{rhBMP2}(\mathrm{ng} / \mathrm{ml})-\quad-2550100$

$\mathrm{IL}-1 \beta(10 \mathrm{ng} / \mathrm{ml})-+++$
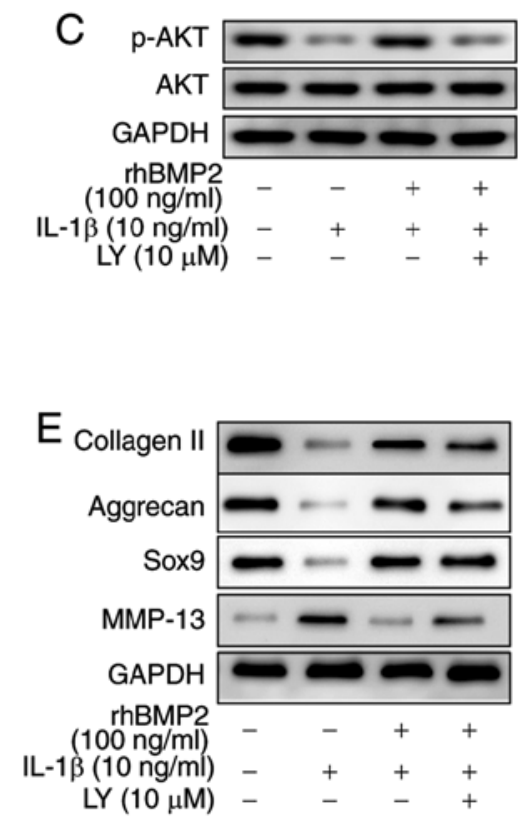
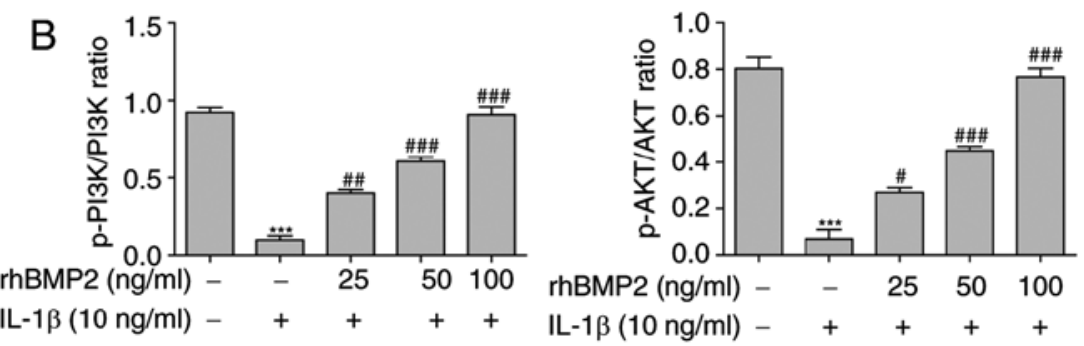
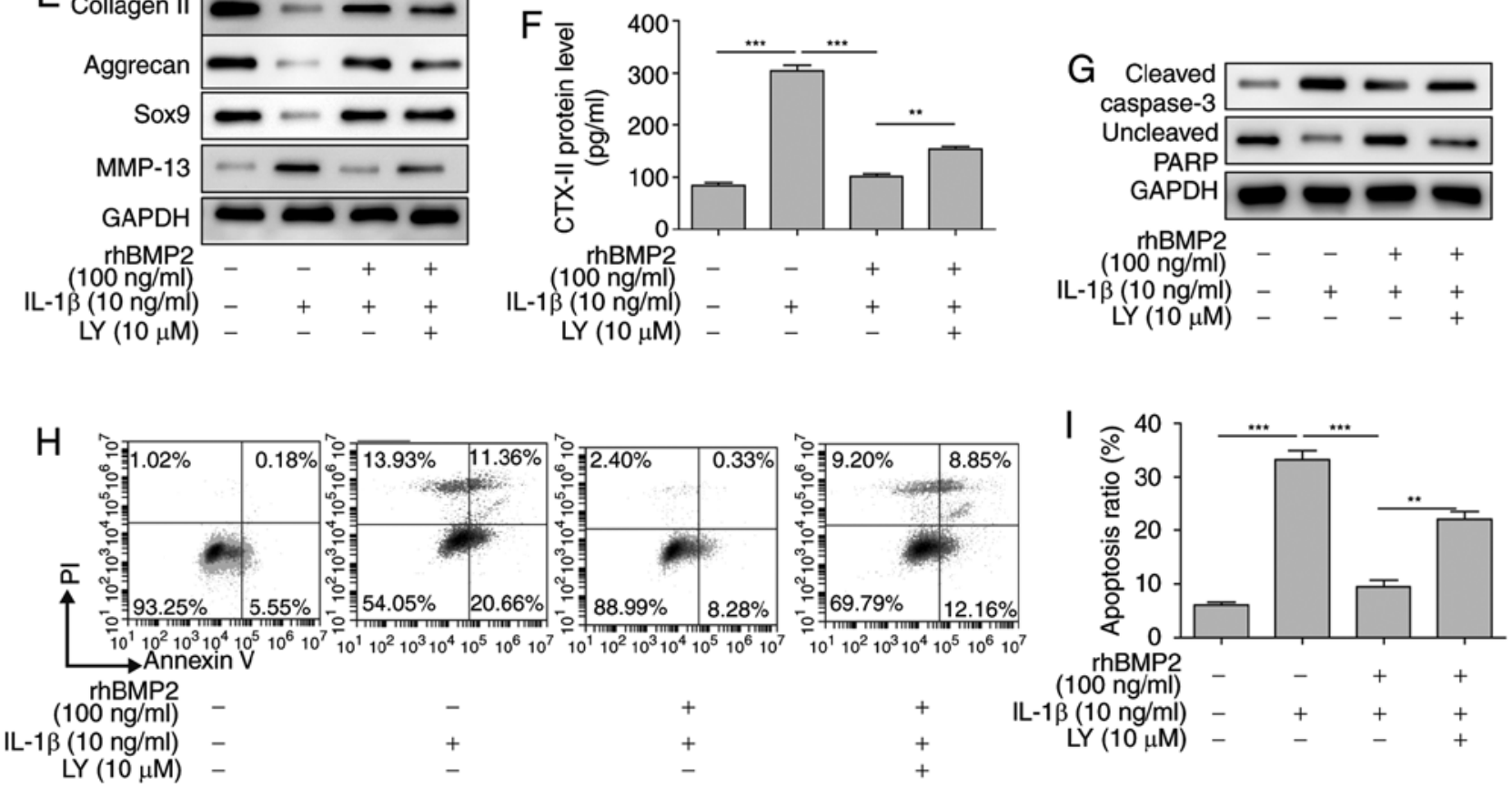

Figure 5. rhBMP2 inhibits the extracellular matrix degradation and apoptosis of NP cells via the PI3K/Akt signaling pathway. (A) Levels of phosphorylation of PI3K and Akt determined by western blot analysis. (B) Quantification of western blot analysis results from part A. (C) Levels of phosphorylated Akt and total Akt in response to IL-1 $\beta$, rhBMP2 and LY294002 treatment as determined by western blot analysis. (D) Quantification of western blot analysis results from part C. (E) Effects of LY294002 treatment on the protein levels involved in the extracellular matrix as determined by western blot analysis. (F) Effects of LY29004 treatment on the level of CTX-II in the supernatant of NP cells determined by ELISA. (G) Levels of apoptosis-associated proteins as determined by western blot analysis. (H) Flow cytometry analysis of cell apoptosis following treatment with combinations of rhBMP2, IL-1 $\beta$ and LY294002. (I) Quantification of flow cytometry results from part $\mathrm{H} .{ }^{* *} \mathrm{P}<0.01 ;{ }^{* * *} \mathrm{P}<0.001$ vs. control group. ${ }^{\#} \mathrm{P}<0.05,{ }^{\# \#} \mathrm{P}<0.01$ and ${ }^{\# \# \|} \mathrm{P}<0.001$ vs. IL-1 1 -treated group. rhBMP2, recombinant human bone morphogenic protein 2; PI3K, phosphoinositide 3-kinase; Akt, protein kinase B; IL, interleukin; LY, LY29004; SOX9, transcription factor SOX9; MMP-13, matrix metalloproteinase 13; CTX-II, C-telopeptide of type II collagen; p-phosphorylated; PI, propidium iodide; NP, nucleus pulposus.

suggest that rhBMP2 attenuated the levels of apoptosis and extracellular matrix degradation of NP cells, potentially via the PI3K/Akt signaling pathway.

\section{Discussion}

IDD is a common chronic disease contributing to lower back pain, and is considered to be one of the most important public health problems worldwide (32). Although a number of trials have been performed to identify treatments for IDD, the situation remains unresolved, owing to the lack of clear understanding of the initiation and pathogenesis of IDD. Considering this, BMP2 was utilized in the present study as a novel promising treatment for IDD via targeting ECM degradation, and proliferation and apoptosis levels of NP cells. Furthermore, the underlying mechanism of BMP2 in treating IDD was examined to provide novel insights on the pathogenesis and treatment of IDD. 
To explore the pathogenesis of IDD, a needle puncture model in rats was constructed. The results demonstrated that AAV2-BMP2 injection significantly alleviated the fibrous ring rupture and glycoproteins degradation of IDD in a dosedependent manner, as determined by MRI and histological analyses. Leckie et al (33) also identified that BMP2 pretreatment delayed the onset of degenerative changes, as measured by MRI, histological examination and serum biomarkers. BMP2 is one of a group of BMPs belonging to the TGF- $\beta$ family, and serves critical roles in skeletal development and repair (34). Concurrently, BMP2 may also directly promote the synthesis of collagen via stimulating chondrocytes (35). Clinical data suggested that rhBMP2 may also promote spinal fusion and bone healing (23). Due to this function, a significant inhibition in the degradation of collagen and significant decrease in the production of CTX-II was observed following AAV2-BMP2 injection in the present study. In addition, the MMP-13 level was also significantly downregulated following treatment with AAV2-BMP2 in IDD. These results suggest that BMP2 may promote the synthesis of collagen and inhibit the degradation of collagen in ECM against IDD. Concomitantly, aggrecan, an additional component of ECM, and its transcription factor SOX9 (36), were significantly upregulated following AAV2-BMP2 injection in IDD. These data indicate that BMP2 upregulated the level of aggrecan via promotion of the transcription of SOX9 to attenuate the pathology of IDD. Previous studies have indicated that certain pro-inflammatory cytokines, including TNF- $\alpha$, may stimulate the degradation of the ECM of intervertebral discs $(37,38)$. In the present study, it was demonstrated that BMP2 inhibited TNF- $\alpha$ and IL-6 expression levels and increased IL-10 expression, suggesting that BMP2 may alleviate intervertebral disc ECM degradation by regulating inflammatory factors. Considering these results, we hypothesize that BMP2 may promote the synthesis of collagen and aggrecan, and inhibit the degradation of ECM, to inhibit the pathogenesis of IDD.

To additionally reveal the underlying mechanism of BMP2 in alleviating IDD (5), the molecular mechanism of BMP2 in NP cells was explored in vitro. As the secretion of inflammatory cytokines is a common characteristic of IDD, IL-1 $\beta$ was used to induce an IDD cell model in NP cells in the present study. Subsequently, it was identified that pretreatment with rhBMP2 significantly inhibited the apoptosis levels of NP cells. In addition, rhBMP2 pretreatment also significantly upregulated the levels of collagen-II, aggrecan and SOX9, but downregulated the levels of MMP-13 and CTX-II in NP cells. The results were consistent with the results identified in the IDD model of the present study. Previous studies have demonstrated that the synthesis of aggrecan was closely correlated with the phosphorylation of the PI3K/Akt signaling pathway, which also performs crucial roles in cell growth, proliferation, migration and invasion $(26,39)$. Inhibiting the PI3K signaling pathway leads to a decrease in the expression levels of aggrecan, collagen II and SOX9 $(26,40,41)$. In the present study, no significantly differences in the levels of total Akt and PI3K following treatment with IL-1 $\beta$ alone or in combination with rhBMP2 were observed, but the levels of phosphorylation of $\mathrm{PI} 3 \mathrm{~K}$ and Akt were significantly decreased following treatment with IL-1 $\beta$, and rhBMP treatment markedly upregulated the levels of phosphorylation of PI $3 \mathrm{~K}$ and Akt in a dose-dependent manner. The PI3K inhibitor LY29400 significantly attenuated the effects of rhBMP2 on the phosphorylation of PI3K and Akt, and reversed the effects on the levels of apoptosis and IDD-associated proteins induced by rhBMP2 in IL-1 $\beta$-treated NP cells. Cheng et al (42) also documented that PI3K/Akt regulated the expression levels of aggrecan and SOX9 in NP cells. Taken together, all of these data indicate that BMP2 may alleviate the levels of apoptosis and ECM degradation in IDD via the PI3K/Akt signaling pathway.

In conclusion, inhibition of ECM degradation and NP cells apoptosis, which are two primary characteristics of IDD, may delay the process of IDD. Therefore, BMP2 may provide a potential strategy for IDD treatment by increasing the production of collagen II, aggrecan and SOX9, decreasing the levels of CTX-II and MMP-13 in the PI3K/Akt signaling pathway and increasing the survival of NP cells. The data of the present study indicate that BMP2 may serve as a promising therapeutic method in treating IDD. However, the safety of BMP2 in clinical applications requires additional examination.

\section{Acknowledgements}

Not applicable.

\section{Funding}

The present study was supported by National Natural Science Foundation of China (grant no. 81401842).

\section{Availability of data and materials}

All data generated or analyzed during this study are included in this published article.

\section{Authors' contributions}

YT is the guarantor of integrity of the entire study, and was responsible for the study concept, design and manuscript preparation. $\mathrm{XY}$ was responsible for study conception and literature research. ZD performed experiments and data acquisition. YW conducted data analysis and statistical analysis. GL performed manuscript drafting and conducted analysis and interpretation of data. All authors read and approved the final manuscript.

\section{Ethics approval and consent to participate}

The experimental procedures were approved by the Second Xiangya Hospital, Central South University (Changsha, China). All the procedures in the present study involving animals and their care were performed in accordance with the Guidelines for the Care and Use of Laboratory Animals of the Second Xiangya Hospital, Central South University.

\section{Patient consent for publication}

Not applicable.

\section{Competing interests}

The authors declare that they have no competing interests. 


\section{References}

1. Shah AM, Kwon SYJ, Chan WC and Chan D: Intervertebral disc degeneration. Cartilage, Springer, pp229-261, 2017.

2. Lim KZ, Daly CD, Ghosh P, Jenkin G, Oehme D, Cooper-White J, Naidoo T and Goldschlager T: Ovine lumbar intervertebral disc degeneration model utilizing a lateral retroperitoneal drill bit injury. J Vis Exp: May 23, 2017. doi: 10.3791/55753.

3. Muriuki MG, Havey RM, Voronov LI, Carandang G, Zindrick MR, Lorenz MA, Lomasney L and Patwardhan AG: Effects of motion segment level, Pfirrmann intervertebral disc degeneration grade and gender on lumbar spine kinematics. J Orthop Res 34: 1389-1398, 2016.

4. Phillips KL, Cullen K, Chiverton N, Michael AL, Cole AA Breakwell LM, Haddock G, Bunning RA, Cross AK and Le Maitre CL: Potential roles of cytokines and chemokines in human intervertebral disc degeneration: Interleukin-1 is a master regulator of catabolic processes. Osteoarthritis Cartilage 23: 1165-1177, 2015.

5. Wang S, Liu C, Sun Z, Yan P, Liang H, Huang K, Li C and Tian J: IL-1 $\beta$ increases asporin expression via the NF- $\mathrm{BB}$ p65 pathway in nucleus pulposus cells during intervertebral disc degeneration. Sci Rep 7: 4112, 2017.

6. Nelson AE and Jordan JM: Osteoarthritis: Epidemiology and classification. In: Rheumatology. Hochberg M, Silman AJ, Smolen J, Weinblatt $M$ and Weisman $M$ (eds). 6th edition. Elsevier, Philadelphia PA, pp1433-1440, 2014.

7. Segar A, Urban J and Fairbank JCT: Adipokines and the intervertebral disc: A biochemical link exists between obesity, intervertebral disc degeneration and low back pain. Spine J 16 (Suppl): S225, 2016.

8. Dong S, Peng L, Jie C, Ma Z, Liu J and Qin T: Correlation between intervertebral disc degeneration, paraspinal muscle atrophy, and lumbar facet joints degeneration in patients with lumbar disc herniation. BMC Musculoskeletal Disorders 18: 167, 2017.

9. Sakai D and Grad S: Advancing the cellular and molecular therapy for intervertebral disc disease. Adv Drug Deliv Rev 84: $159-171,2015$

10. Wang AM, Cao P, Yee A, Chan D and Wu EX: Detection of extracellular matrix degradation in intervertebral disc degeneration by diffusion magnetic resonance spectroscopy. Magn Reson Med 73: 1703-1712, 2015.

11. Gou S, Oxentenko SC, Eldrige JS, Xiao L, Pingree MJ, Wang Z, Perez-Terzic C and Qu W: Stem cell therapy for intervertebral disk regeneration. Am J Phys Med Rehabil 93 (11 Suppl 3): S122-S131, 2014.

12. Le Maitre CL, Pockert A, Buttle DJ, Freemont AJ and Hoyland JA: Matrix synthesis and degradation in human intervertebral disc degeneration. Biochem Soc Trans 35: 652-655, 2007.

13. Huang X, Deng X, Xu H, Wu S, Yuan L, Yang Z, Yang Y and Deng H: Identification of a novel mutation in the COL2A1 gene in a Chinese Family with Spondyloepiphyseal dysplasia Congenita PLoS One 10: e0127529, 2015.

14. Barathouari M, Sarrabay G, Gatinois V, Fabre A, Dumont B, Genevieve D and Touitou I: Mutation update for COL2A1 gene variants associated with type II collagenopathies. Hum Mutat 37: $7-15,2016$

15. Xu YQ, Zhang ZH, Zheng YF and Feng SQ: Dysregulated miR-133a mediates loss of type II collagen by directly targeting matrix metalloproteinase 9 (MMP9) in human intervertebral disc degeneration. Spine (Phila Pa 1976) 41: E717-E724, 2016.

16. Xin L, Wu Z, Qu Q, Wang R, Tang J and Chen L: Comparative study of CTX-II, Zn2+, and Ca2+ from the urine for knee osteoarthritis patients and healthy individuals. Medicine (Baltimore) 96: e7593, 2017.

17. Yang SD, Yang DL, Sun YP, Wang BL, Ma L, Feng SQ and Ding WY: $17 \beta$-estradiol protects against apoptosis induced by interleukin-1 $\beta$ in rat nucleus pulposus cells by down-regulating MMP-3 and MMP-13. Apoptosis 20: 348-357, 2015.

18. Miller SL, Coughlin DG, Waldorff EI, Ryaby JT and Lotz JC: Pulsed electromagnetic field (PEMF) treatment reduces expression of genes associated with disc degeneration in human intervertebral disc cells. Spine J 16: 770-776, 2016.

19. Ye S, Ju B, Wang H and Lee KB: Bone morphogenetic protein-2 provokes interleukin-18-induced human intervertebral disc degeneration. Bone Joint Res 5: 412-418, 2016.

20. Khosla S, Westendorf JJ and Oursler MJ: Building bone to reverse osteoporosis and repair fractures. J Clin Invest 118: $421-428,2008$
21. McKay WF, Peckham SM and Badura JM: A comprehensive clinical review of recombinant human bone morphogenetic protein-2 (INFUSE Bone Graft). Int Orthop 31: 729-734, 2007.

22. Sobajima S, Shimer AL, Chadderdon RC, Kompel JF, Kim JS, Gilbertson LG and Kang JD: Quantitative analysis of gene expression in a rabbit model of intervertebral disc degeneration by real-time polymerase chain reaction. Spine J 5: 14-23, 2005.

23. Bougioukli S, Jain A, Sugiyama O, Tinsley BA, Tang AH, Tan MH, Adams DJ, Kostenuik PJ and Lieberman JR: Combination therapy with BMP-2 and a systemic RANKL inhibitor enhances bone healing in a mouse critical-sized femoral defect. Bone 84: 93-103, 2016

24. Zheng Y, Wang X, Wang H, Yan W, Zhang Q and Chang X: Bone morphogenetic protein 2 inhibits hepatocellular carcinoma growth and migration through downregulation of the PI3K/AKT pathway. Tumor Biol 35: 5189-5198, 2014

25. Qu Z, Guo S, Fang G, Cui Z and Ying L: AKT pathway affects bone regeneration in nonunion treated with umbilical cord-derived mesenchymal stem cells. Cell Biochem Biophys 71: 1543-1551,2015.

26. Ouyang ZH, Wang WJ, Yan YG, Wang B and Lv GH: The PI3K/Akt pathway: A critical player in intervertebral disc degeneration. Oncotarget 8: 57870-57881, 2017.

27. Bonino F, Heermann KH, Rizzetto M and Gerlich WH: Hepatitis delta virus: Protein composition of delta antigen and its hepatitis B virus-derived envelope. J Virol 58: 945-950, 1986.

28. Issy AC, Castania V, Castania M, Salmon CE, Nogueira-Barbosa MH, Bel ED and Defino HL: Experimental model of intervertebral disc degenerationby needle puncture in Wistar rats. Braz J Med Biol Res 46: 235-244, 2013.

29. Li Z, Shen J, Wu WK, Yu X, Liang J, Qiu G and Liu J: Leptin induces cyclin D1 expression and proliferation of human nucleus pulposus cells via JAK/STAT, PI3K/Akt and MEK/ERK pathways. PLoS One 7: e53176, 2012.

30. Li Z, Shen J, Wu WK, Yu X, Liang J, Qiu G and Liu J: The role of leptin on the organization and expression of cytoskeleton elements in nucleus pulposus cells. J Orthop Res 31: 847-857, 2013.

31. Livak KJ and Schmittgen TD: Analysis of relative gene expression data using real-time quantitative PCR and the 2(-Delta Delta C(T)) method. Methods 25: 402-408, 2001.

32. Wang XF, Zhang AP, Sun ZY, Liu C, Kuang LH and Tian JW: Expression of NF- $\kappa \mathrm{B}$ in a degenerative human intervertebral disc model. Zhonghua Yi Xue Za Zhi 97: 1324-1329, 2017 (In Chinese).

33. Leckie SK, Bechara BP, Hartman RA, Sowa GA, Woods BI, Coelho JP, Witt WT, Dong QD, Bowman BW, Bell KM, et al: Injection of AAV2-BMP2 and AAV2-TIMP1 into the nucleus pulposus slows the course of intervertebral disc degeneration in an in vivo rabbit model. Spine J 12: 7-20, 2012.

34. Leung VY, Zhou L, Tam WK, Sun Y, Lv F, Zhou G and Cheung KMC: Bone morphogenetic protein -2 and -7 mediate the anabolic function of nucleus pulposus cells with discrete mechanisms. Connect Tissue Res 58: 573-585, 2017.

35. Lafont JE, Poujade FA, Pasdeloup M, Neyret $P$ and Mallein-Gerin F: Hypoxia potentiates the BMP-2 driven COL2A1 stimulation of human articular chondrocytes via p38 MAPK. Osteoarthritis Cartilage 24: 856-867, 2016.

36. Kumar A, Oz MB, Elayyan J, Reich E, Binyamin M, Kandel L, Liebergall M, Steinmeyer J and Lefebvre V: SOX9 acetylation reduces aggrecan expression in adult human chondrocytes. Osteoarthritis and Cartilage 24: S154, 2016.

37. Xu K, Wang X, Zhang Q, Liang A, Zhu H, Huang D, Li C and Ye W: Sp1 downregulates proinflammatory cytokine-induced catabolic gene expression in nucleus pulposus cells. Mol Med Rep 14: 3961-3968, 2016.

38. Walter BA, Purmessur D, Moon A, Occhiogrosso J, Laudier DM, Hecht AC and Iatridis JC: Reduced tissue osmolarity increases TRPV4 expression and pro-inflammatory cytokines in intervertebral disc cells. Eur Cell Mater 32: 123-136, 2016.

39. Cantley LC: The phosphoinositide 3-kinase pathway. Science 296: 1655-1657, 2002.

40. Kita K, Kimura T, Nakamura N, Yoshikawa H and Nakano T: PI3K/Akt signaling as a key regulatory pathway for chondrocyte terminal differentiation. Genes Cells 13: 839-850, 2008.

41. Li N, Cui J, Duan X, Chen H and Fan F: Suppression of type I collagen expression by miR-29b via PI3K, Akt, and Sp1 pathway in human Tenon's fibroblasts. Invest Ophthalmol Vis Sci 53: 1670-1678, 2012

42. Cheng CC, Uchiyama Y, Hiyama A, Gajghate S, Shapiro IM and Risbud MV: PI3K/AKT regulates aggrecan gene expression by modulating Sox9 expression and activity in nucleus pulposus cells of the intervertebral disc. J Cell Physiol 221: 668-676, 2009. 